\title{
Sommaire
}

Avant-propos : par Paul Rigny ............................. 9

Préface : par Bernard Bigot ................................. 11

Introduction : La simplexité

par Alain Berthoz

Partie 1

La quête et les limites de la performance

Chapitre 1 : Optimisation des performances, complexité des systèmes

et confrontation aux limites

par Jean-François Toussaint

Chapitre 2 : La fabrique des surhommes:

corps entraîné, corps dopé, corps augmenté

par Isabelle Queval

Chapitre 3 : Technologie et performance sportive par D'après la conférence de Denis Masseglia

Chapitre 4 : Comprendre la physico-chimie par la plongée sous-marine. Comprendre la plongée sous-marine par la physico-chimie par Pierre Letellier

\section{Partie 2}

Les molécules de la performance

Chapitre 5 : Effets de l'exercice physique et de l'entraînement sur la neurochimie cérébrale : effets sur la performance et la santé mentale par Charles-Yannick Guezennec 
Chapitre 6 : La traque aux molécules dopantes par Jean-Luc Veuthey

Chapitre 7 : Les molécules de la performance par Marie-Florence Grenier-Loustalot

\section{Partie 3 \\ Les matériaux de la performance}

Chapitre 8 : Les matériaux composites dans le sport

par Yves Rémond et Jean-François Caron

Chapitre 9 : Performance d'un ski de course : structure composite et glisse sur neige par Nicolas Puget

Chapitre 10 : Revêtements complexes antifriction pour les composants moteurs automobiles. De la F1 à la grande série par Claude Lory

Chapitre 11 : Des textiles pour sportifs. Apport de la chimie pour améliorer confort et performances par Fabien Roland 239

Clossaire 255

Crédits photographiques 257 